\title{
Research Article \\ Second Order Sliding Mode Control of the Coupled Tanks System
}

\author{
Fayiz Abu Khadra' ${ }^{1}$ and Jaber Abu Qudeiri ${ }^{2}$ \\ ${ }^{1}$ Faculty of Engineering, King Abdulaziz University, Rabigh 21911, Saudi Arabia \\ ${ }^{2}$ FARCAMT, Advanced Manufacturing Institute, King Saud University, Riyadh 11421, Saudi Arabia \\ Correspondence should be addressed to Jaber Abu Qudeiri; jqudeiri@ksu.edu.sa
}

Received 7 March 2015; Revised 4 April 2015; Accepted 5 April 2015

Academic Editor: Qingling Zhang

Copyright ( 2015 F. Abu Khadra and J. Abu Qudeiri. This is an open access article distributed under the Creative Commons Attribution License, which permits unrestricted use, distribution, and reproduction in any medium, provided the original work is properly cited.

\begin{abstract}
Four classes of second order sliding mode controllers (2-SMC) have been successfully applied to regulate the liquid level in the second tank of a coupled tanks system. The robustness of these classes of 2-SMC is investigated and their performances are compared with a first order controller to show the merits of these controllers. The effectiveness of these controllers is verified through computer simulations. Comparison between the controllers is based on the time domain performance measures such as rise time, settling time, and the integral absolute error. Results showed that controllers are able to regulate the liquid level with small differences in their performance.
\end{abstract}

\section{Introduction}

The control of liquid level in multiple connected tanks by controlling the liquid flow is a typical nonlinear control problem in the field of process control. It is present in many industrial processes. Many researchers around the world have attempted the design and implementation of controllers for the liquid level of a coupled tanks system. Some of the controllers used to control coupled tanks systems include Proportional-Integral-Derivative (PID) type controllers [1], a parallel structure of fuzzy PID control systems [2], a nonlinear constrained predictive algorithms based on feedback linearization control [3], and fractional PID controller [4].

Sliding mode control is an efficient method for robust control of uncertain systems [5-7]. The basic idea of the first order sliding mode control (1-SMC) is to let the system converge towards a selected surface and then to stay there in spite of uncertainties and disturbances. The 1-SMC method can be designed by performing two steps. The first step is to select an appropriate sliding surface to constrain the state trajectory on it. The second step includes designing of a discontinuous control law to force the system state to reach the designed surface preferably in finite time. 1-SMC requires sliding variable relative degree (the relative degree is defined as the order of the derivative of the controlled variable, in which the control input appears explicitly) to be equal to one with respect to the control input which limits the choice of the sliding variable. The 1-SMC is also used to regulate the liquid level. An input-dependent sliding surface has been used in [8] to regulate the liquid level in a coupled tanks system. A sliding mode controller, which has a state varying sliding surface parameter, has been designed in [9]. A neuro-fuzzysliding mode controller using nonlinear sliding surface has been proposed in [10].

In addition to the restriction regarding the relative degree, 1-SMC also has the drawback of chattering due to high switching frequency of the control. The drawbacks of 1SMC can be successfully eliminated by the use of higher order sliding mode controllers (HOSMC). HOSMC force the sliding variable and its successive derivatives to zero. There is no restriction on the relative degrees. As the high frequency control switching is pushed in the higher derivative of the sliding variable, chattering is significantly reduced. Another feature of HOSMC is that the detailed mathematical model of the plant is not required. The most widely used HOSMC are second order sliding mode controllers (2-SMC). Examples of 
2-SMC are widely used twisting controllers and its modified variant the super twisting controllers, the quasi-continuous controllers, the suboptimal control algorithm, and the control algorithm with prescribed convergence law.

Khan and Spurgeon [11] applied a second order sliding mode control idea to control a coupled tank system.

The super twisting and adaptive super twisting control algorithms are developed for the two-spacecraft formation flying system in [12]. A 2-SMC is proposed for second order uncertain plants using equivalent control approach to improve the performance of control systems in [13]. A discrete integral sliding mode controller based on composite nonlinear feedback method to improve the transient performance of uncertain systems is proposed in [14]. A second order sliding mode controller using nonlinear sliding surface to guarantee stability as well as to enhance the transient performance of uncertain linear systems with parametric uncertainty has been proposed in [15]. An adaptive second order sliding mode controller with a nonlinear sliding surface that consists of a gain matrix having a variable damping ratio has been presented in [16]. A higher order sliding mode control algorithm is described for a class of uncertain multiinput multioutput nonlinear systems and the developed algorithm was applied on a hovercraft vessel control [17].

Despite the many existing publications related to the 2SMC, there is a lack of articles that compare performance of different types of 2-SMC in water level of the second tank in the coupled tanks system from one side and between them and the first order controller from the other side. Moreover, to the best of authors' knowledge, there is no published work concerning the 2-SMC especially, which contains the detailed analysis of the time domain control measures and the tracking performance of the well-known controller.

In this paper, robustness of four classes of 2-SMC, namely, twisting (TA), the super twisting (STC), prescribed convergence law controller (PCL), and the quasi-continuous controller (QCC), to regulate the water level of the second tank in the coupled tanks system is introduced and their performances are compared. Moreover, the performances of the four controllers mentioned above are compared with a first order controller to show the merits of these controllers.

The remaining structure of this paper is as follows. In the next section, the dynamic model of the coupled tank system will be explained. Section 3 briefly provides the basics of the 1-SMC controller and the 2-SMC. In Section 4, 2-SMC will be described briefly. In Section 5, the simulation results from the application of the controller will be presented and discussed. Finally, Section 6 includes the concluding remarks based on the results obtained.

\section{Mathematical Modeling of the Coupled Tanks System}

Figure 1 shows a schematic diagram of the two-coupled tanks system. The tanks system consists of two connected tanks. A pump supplies the water into the first tank. The second tank is filled from the first tank via a connecting pipe. An outlet is located at the bottom of the second tank to change the output

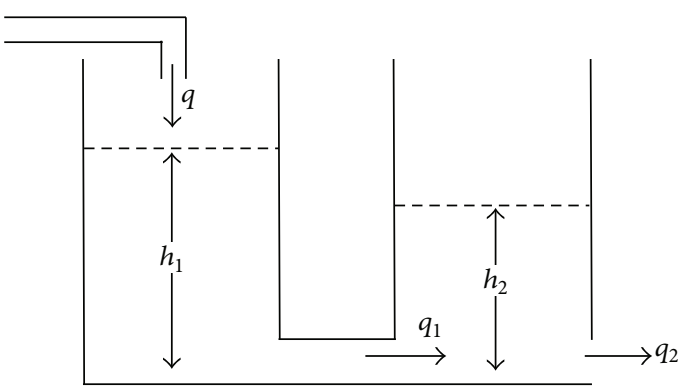

FIgURE 1: A schematic diagram of the two-coupled tanks system.

flow $q_{2}$. The mathematical model of the coupled tanks system is nonlinear and can be derived as follows.

Applying the flow balance equation for Tanks 1 and 2,

$$
\begin{aligned}
& \frac{d h_{1}}{d t}=\frac{1}{A}\left(q-q_{1}\right), \\
& \frac{d h_{2}}{d t}=\frac{1}{A}\left(q_{1}-q_{2}\right) .
\end{aligned}
$$

In (1) $q_{1}$ and $q_{2}$ are defined as

$$
\begin{aligned}
& q_{1}=a_{1} \sqrt{2 g\left(h_{1}-h_{2}\right)} \text { for } h_{1}>h_{2}, \\
& q_{2}=a_{2} \sqrt{2 g h_{2}} \text { for } h_{2}>0,
\end{aligned}
$$

where $h_{1}$ and $h_{2}$ are the water level in Tank 1 and Tank 2, respectively. $q$ is the inlet flow rate; $q_{1}$ is the flow rate from Tank 1 to Tank 2. $A$ is the cross section area for both tanks, $a_{1}$ is the area of pipe connecting the two tanks, $a_{2}$ is the area of the outlet, and $g$ is the constant of gravity. If the inlet flow $q$ is selected as input and the liquid level $h_{2}$ in the second tank as output, the system can be considered as a single input single output system (SISO). The two tanks system can be modeled by the following two differential equations:

$$
\begin{aligned}
& \frac{d h_{1}}{d t}=-k_{1} \operatorname{sign}\left(h_{1}-h_{2}\right) \sqrt{\left|h_{1}-h_{2}\right|}+\frac{q}{A}, \\
& \frac{d h_{2}}{d t}=k_{1} \operatorname{sign}\left(h_{1}-h_{2}\right) \sqrt{\left|h_{1}-h_{2}\right|}-k_{2} \sqrt{h_{2}} .
\end{aligned}
$$

The parameters $k_{1}$ and $k_{2}$ are defined by

$$
\begin{aligned}
& k_{1}=\frac{a_{1} \sqrt{2 g}}{A}, \\
& k_{2}=\frac{a_{2} \sqrt{2 g}}{A} .
\end{aligned}
$$

Note that $q$ is always positive, which means that the pump can pump water into the tank $(q \geq 0)$. At equilibrium, for constant water level set point, the derivatives of the water levels with respect to time in the two tanks must be zero so that the following condition can be written:

$$
\frac{d h_{1}}{d t}=\frac{d h_{2}}{d t}=0
$$


Therefore, using (6) in (3) and (4), the following algebraic relationships hold:

$$
\begin{gathered}
-k_{1} \operatorname{sign}\left(h_{1}-h_{2}\right) \sqrt{\left|h_{1}-h_{2}\right|}+\frac{q}{A}, \\
k_{1} \operatorname{sign}\left(h_{1}-h_{2}\right) \sqrt{\left|h_{1}-h_{2}\right|}-k_{2} \sqrt{h_{2} .}
\end{gathered}
$$

The equilibrium flow rate $q$ can be calculated as

$$
q=-A k_{1} \operatorname{sign}\left(h_{1}-h_{2}\right) \sqrt{\left|h_{1}-h_{2}\right|} .
$$

To satisfy the constraint on the input flow rate, the term $k_{1} \operatorname{sign}\left(h_{1}-h_{2}\right) \geq 0$, which implies $h_{1} \geq h_{2}$. Then the dynamics model can be written as

$$
\begin{aligned}
& \frac{d h_{1}}{d t}=-k_{1} \sqrt{h_{1}-h_{2}}+k_{2} \sqrt{h_{2},} \\
& \frac{d h_{2}}{d t}=-k_{1} \sqrt{h_{1}-h_{2}}-2 k_{2} \sqrt{h_{2}}+\frac{1}{A} u .
\end{aligned}
$$

Using the following transformation,

$$
\begin{aligned}
& x_{1}=h_{1}, \\
& x_{2}=-k_{1} \sqrt{h_{1}}+k_{2} \sqrt{h_{1}-h_{2}} .
\end{aligned}
$$

(9) can be written as

$$
\begin{aligned}
\dot{x}_{1} & =x_{2} . \\
\dot{x}_{2} & =f(x, t)+b(x, t) u \\
y & =x_{1} .
\end{aligned}
$$

It can be easily checked that $f(x, t)$ and $b(x, t)$ in (11) have the following form:

$$
\begin{aligned}
& f(x, t)=\frac{k_{1} k_{2}}{2}\left(\frac{\sqrt{h_{2}}}{\sqrt{h_{1}-h_{2}}}-\frac{\sqrt{h_{1}-h_{2}}}{\sqrt{h_{2}}}\right)+\frac{k_{1}^{2}}{2}-k_{2}^{2}, \\
& b(x, t)=\frac{k_{2}}{2 A} \frac{1}{\sqrt{h_{1}-h_{2}}} .
\end{aligned}
$$

\section{Sliding Mode Control}

3.1.1-SMC. The dynamics of a second order nonlinear system can be written as

$$
\ddot{x}=f(x)+b(x) u,
$$

where $x(t)$ is the state and $u(t)$ is the control input vectors, respectively. $f(x, t)$ and $b(x, t)$ are unknown nonlinear functions of time and states. The functions $f(x)$ and $b(x)$ are not exactly known with upper bounded uncertainties. The control problem let the state $x$ track a specified time-varying state $x_{d}$. The required sliding surface to control this system can be defined as

$$
S=\dot{e}+\lambda e .
$$

$e=x-x_{r}$ is an error vector and $\lambda$ is a constant parameter, which dictated the slope of the sliding surface. Differentiating (16), and inserting (15), and then putting $\dot{S}=0$ and solving for $u$ result in the following control law:

$$
\widehat{u}=-\widehat{f}(\dot{x}, x)+\ddot{x}_{d}+\lambda \dot{\hat{x}}
$$

and define the control law

$$
u=\widehat{u}-k \operatorname{sign}(s),
$$

where $\lambda$ and $k$ are strictly positive constant. $k$ can be selected high to cope with uncertainty. A sufficient condition for the convergence to a sliding mode is

$$
\frac{1}{2} \frac{d}{d t^{2}} s^{2} \leq-\eta|s| .
$$

$\eta$ is a positive constant. Equation (18) is called reaching or sliding condition. The signum function in (17) is defined as

$$
\operatorname{sign}(x)= \begin{cases}-1 & x<0 \\ 0 & x=0 \\ 1 & x>0\end{cases}
$$

Commonly in 1-SMC, to avoid chattering, the signum function is replaced by a smooth function to create a boundary layer around the sliding surface. For example, a saturation function can be used and is given by

$$
\operatorname{sat}(\sigma)= \begin{cases}\operatorname{sign}(\sigma) & \text { if }|\sigma|>\varphi \\ \frac{\sigma}{\varphi} & \text { if }|\sigma| \leq \varphi .\end{cases}
$$

In (22) $\varphi$ is a positive constant which defines the thickness of the boundary layer.

3.2. 2-SMC. 2-SMC control is a subset of HOSMC. It differs from the 1-SMC by including the first order derivative of the sliding variable while maintaining the same robustness and performance as that of the 1-SMC. $\sigma \in \mathbb{R}$ in an output of (15) to be exactly stabilized in finite time at $\sigma=0, u \in \mathbb{R}$ is the control input, and $x \in \mathbb{R}^{n}$ is the state. If the output $\sigma$ has a fixed and known relative degree $r \in \mathbb{R}^{n}$, then, for the positive constants $K_{m}, K_{M}$, and $C$, the following inequalities hold globally:

$$
\begin{aligned}
0 & <K_{m} \leq b \leq K_{M}, \\
|f| & \leq C .
\end{aligned}
$$

2-SMC may be considered as controllers for the following differential inclusion [18]:

$$
\ddot{\sigma} \in[-C, C]+\left[K_{m}, K_{M}\right] u .
$$

2-SM controllers allow solving the problem of finite-time stabilization of a black box system as shown in Figure 2. The only information needed from the system is the output. 


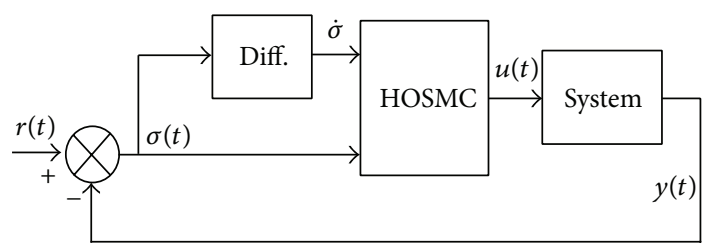

FIGURE 2: Block diagram of a black box controller.

The required derivative can be obtained by using HOSMC arbitrary order differentiator [19]. The real time differentiator has the general form as in the following set of equations. In (24) $f(t)$ represents signal to be differentiated, $k-1$ times. Consider

$$
\begin{aligned}
\dot{z}_{0}= & v_{0}, \\
\dot{z}_{1}= & v_{1} v_{0} \\
= & -\lambda_{k} L^{1 /(k+1)}\left|z_{0}-f(t)\right|^{k /(k+1)} \operatorname{sign}\left(z_{0}-f(t)\right) \\
& +z_{1}, \\
v_{1}= & -\lambda_{k-1} L^{1 / k}\left|z_{1}-v_{0}\right|^{(k-1) / k} \operatorname{sign}\left(z_{1}-v_{0}\right)+z_{2}, \\
\dot{z}_{k-1}= & v_{k-1} \\
= & -\lambda_{1} L^{1 / 2}\left|z_{k-1}-v_{k-2}\right|^{k /(k+1)} \operatorname{sign}\left(z_{k-1}-v_{k-2}\right) \\
& +z_{k}, \\
\dot{z}_{k}= & \lambda_{0} L \operatorname{sign}\left(z_{k}-v_{k-1}\right) .
\end{aligned}
$$

A first order differentiator can be obtained by setting $k=1$ and has the following form:

$$
\begin{aligned}
& \dot{z}_{0}=-\lambda_{1}\left|z_{0}-f(t)\right|^{1 / 2} \operatorname{sign}\left(z_{0}-f(t)\right)+z_{1}, \\
& \dot{z}_{1}=-\lambda_{0} \operatorname{sign}\left(z_{0}-f(t)\right),
\end{aligned}
$$

where $z_{0}$ and $z_{1}$ are the estimation of $f(t)$ and $\dot{f}(t)$, respectively. The parameters $\lambda_{1}=1.5 L^{1 / 2}, \lambda_{0}=1.1 L$, and $L$ are a positive constant to be selected via simulation as recommended in [19].

\section{Controllers Design}

4.1. 1-SM. The procedure described in Section 3.1 will be applied to regulate the output $h_{2}$ to a desired value $H$ for the coupled tanks system. The sliding surface $\sigma$ is selected as

$$
\sigma=\dot{x}_{1}+\lambda\left(x_{1}-H\right) \text {. }
$$

By taking the time derivative of (25), (26) is obtained as follows:

$$
\dot{\sigma}=\ddot{x}_{1}+\lambda \dot{x}_{1} .
$$

Substituting (10) and (11) in (26) yields

$$
\dot{\sigma}=f(x)+b(x) \widehat{u}+\lambda\left(-k_{1} \sqrt{h_{1}}+k_{2} \sqrt{h_{1}-h_{2}}\right) .
$$

On putting $\dot{\sigma}=0$ and solving for $\widehat{u}$,

$$
\widehat{u}=\frac{1}{b(x)}\left[-f(x)-\lambda\left(-k_{1} \sqrt{h_{1}}+k_{2} \sqrt{h_{1}-h_{2}}\right)\right] .
$$

Finally using (18) the control law for $h_{2}$ is computed as

$$
\begin{aligned}
u= & \frac{1}{b(x)}\left[-f(x)-\lambda\left(-k_{1} \sqrt{h_{1}}+k_{2} \sqrt{h_{1}-h_{2}}\right)\right] \\
& -k \operatorname{sign}(\sigma) .
\end{aligned}
$$

4.2. Twisting Controller. Historically, the first proposed 2-SM controller was the twisting controller. It is defined by the following formula

$$
u(t)=-\left(r_{1} \operatorname{sign}(\sigma)+r_{2} \operatorname{sign}(\dot{\sigma})\right)
$$

This controller guarantees the appearance of a 2-sliding mode $\sigma=\dot{\sigma}=0$ attracting the trajectories of the sliding variable dynamics in finite time if $r_{1}$ and $r_{2}$ satisfy the following conditions:

$$
\begin{aligned}
r_{1} & >r_{2}>0, \\
\left(r_{1}+r_{2}\right) K_{m}-C & >\left(r_{1}-r_{2}\right) K_{M}, \\
\left(r_{1}-r_{2}\right) K_{m} & >0 .
\end{aligned}
$$

4.3. Super Twisting Controller. The STC has the advantage over another algorithm in that it does not require the time derivatives of the sliding variables. The algorithm has been developed for the case of a system with relative degree one, in order to avoid chattering and its trajectories on the sliding plane are characterized by twisting around the origin. The merits of the STC are rejection of smooth disturbances of arbitrary shape, tracking of smooth references of arbitrary shape, and the finite-time convergence to the set point. A STC for system, which has relative degree of two, was proposed in [20]. The control law $u(t)$ consists of two terms. The first term is its discontinuous time derivative and the second is a continuous function of the available sliding variable as given below

$$
\begin{gathered}
u(t)=-\lambda|\sigma|^{1 / 2} \operatorname{sign}(\sigma)+u_{1}, \\
\dot{u}_{1}= \begin{cases}-k u & |u|>U_{M} \\
-\alpha \operatorname{sign}(\sigma) & |u| \leq U_{M} .\end{cases}
\end{gathered}
$$

As stated in [19] for $K_{m}>C$ and a sufficiently large $\alpha$ the controller guarantees the appearance of a 2-SMC in system given in (23), which attracts the trajectories in finite time to zero. A sufficient condition for finite time convergence is as follows [19]:

$$
\lambda>\sqrt{\frac{2}{\left(K_{m} \alpha-C\right)}} \frac{\left(K_{m} \alpha+C\right) K_{M}(1+q)}{K_{m}^{2}(1-q)} .
$$



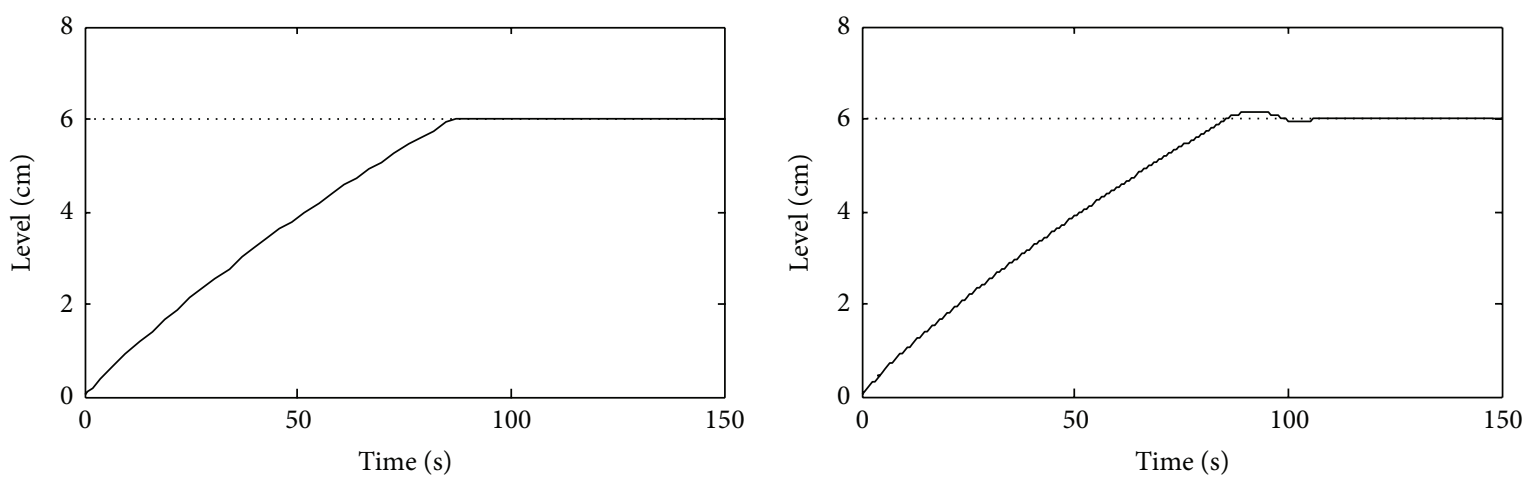

- SMC

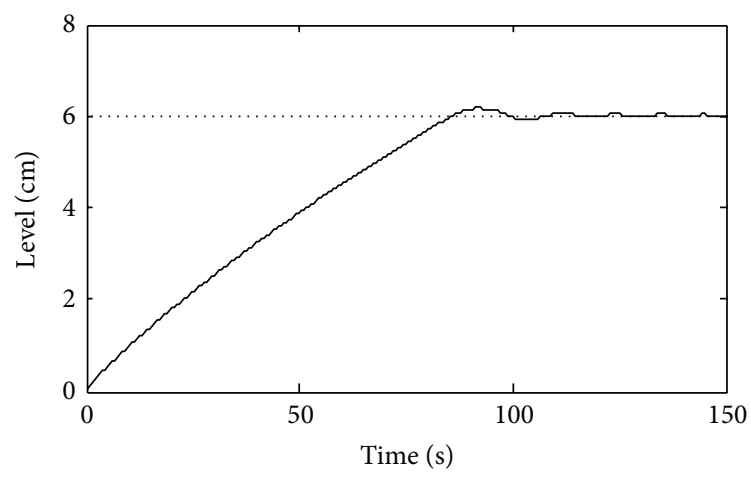

- TA

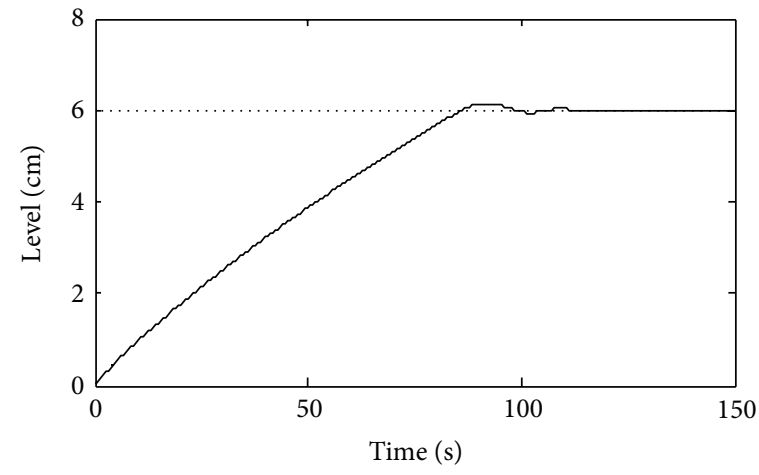

— STC

QCC

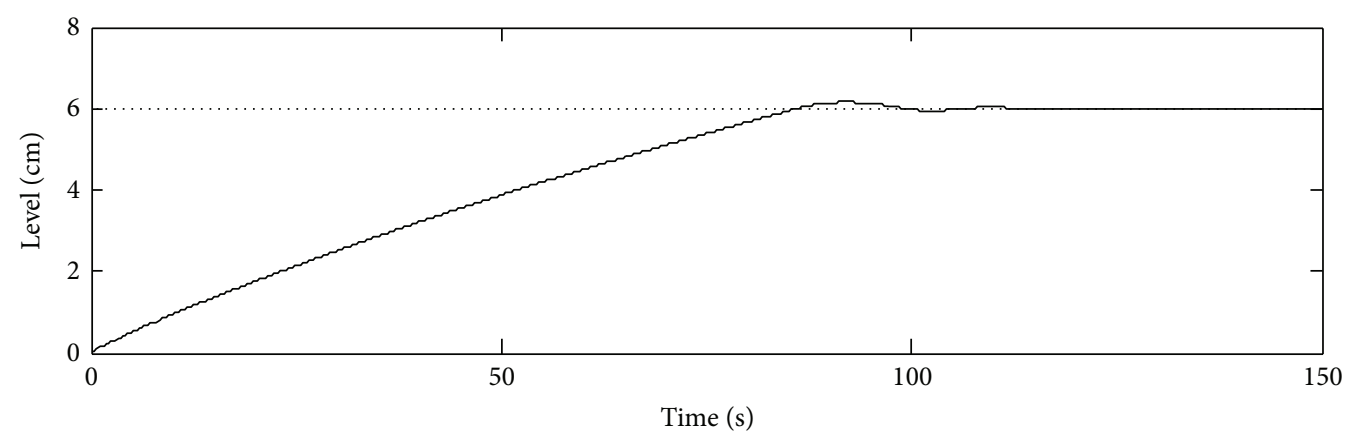

PLC

FIgURE 3: Level tracking test for the different controllers.

4.4. Quasi-Continuous Controller (QCC). The homogeneous quasi-continuous (QCC) controller [21] requires the first order derivative of the sliding surface $\sigma$. With the use of differentiator in (25), the second order quasi-continuous sliding mode controller can be written as

$$
u(t)=-\alpha \frac{z_{1}+\beta|\sigma|^{1 / 2} \operatorname{sign}(\sigma)}{\left|z_{1}\right|+\beta|\sigma|^{1 / 2}} .
$$

The parameters $\alpha$ and $\beta$ in (35) are positive constant to be selected via computer simulation.
4.5. Prescribed Convergence Law Controller. The controller with prescribed convergence law [19] is defined as

$$
u(t)=-\alpha \operatorname{sign}(\dot{\sigma}+\xi(\sigma)),
$$

where $\xi(\sigma)$ is a continuous function smooth everywhere except at $\sigma=0$. It is assumed that the solution of the differential equation $\dot{\sigma}+\xi(\sigma)=0$ converges to 0 in finite time. Choosing $\xi(\sigma)=\beta|\sigma|^{1 / 2} \operatorname{sign}(\sigma)$ yields the controller

$$
u(t)=-\alpha \operatorname{sign}\left(\dot{\sigma}+\beta|\sigma|^{1 / 2} \operatorname{sign}(\sigma)\right) .
$$



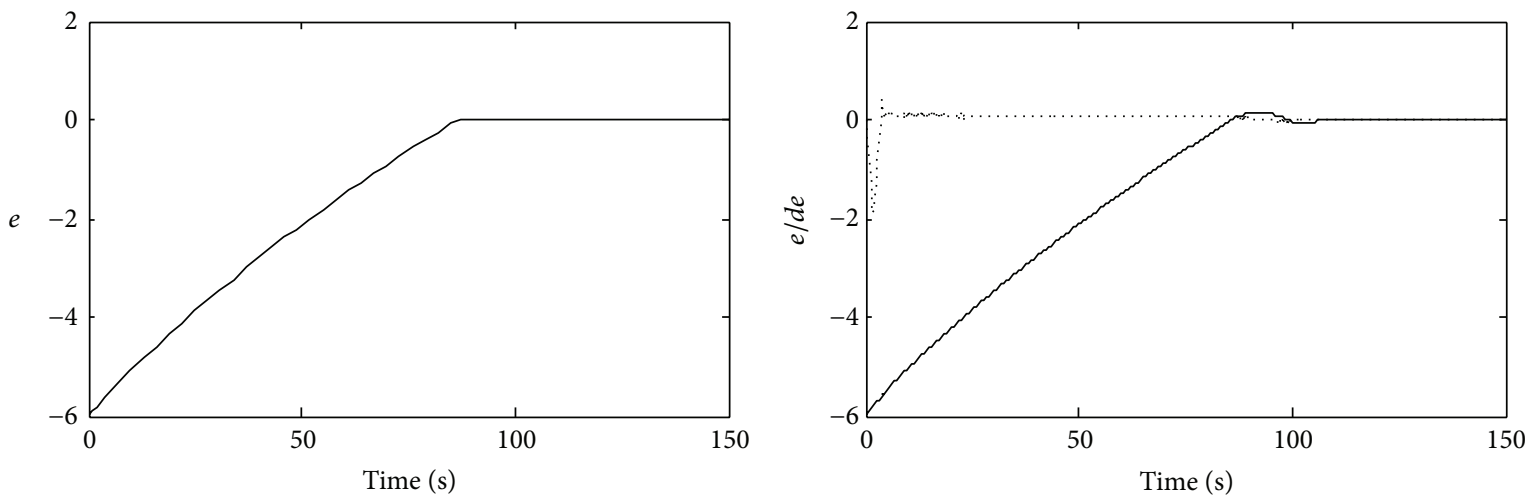

$-e(\mathrm{SMC})$

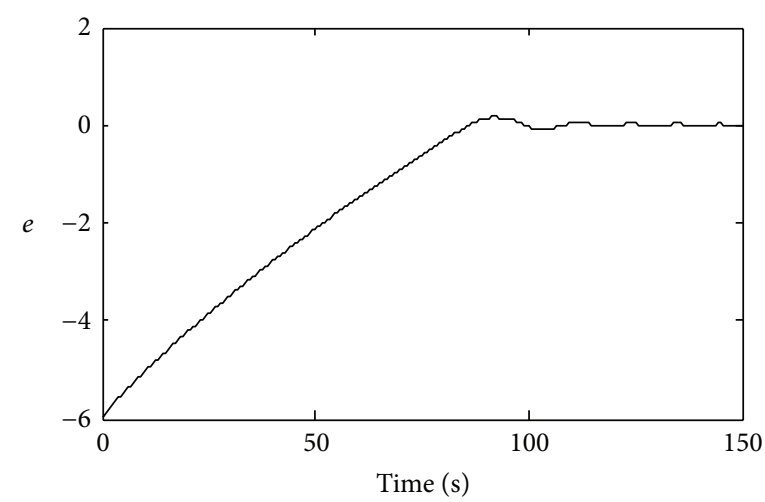

$-e(\mathrm{TA})$

.... $d e(\mathrm{TA})$

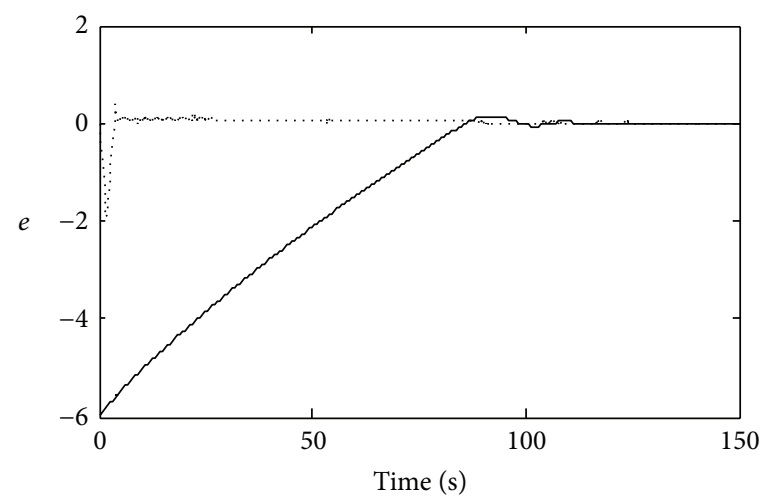

— STC

- $e(\mathrm{QCC})$

..... de (QCC)

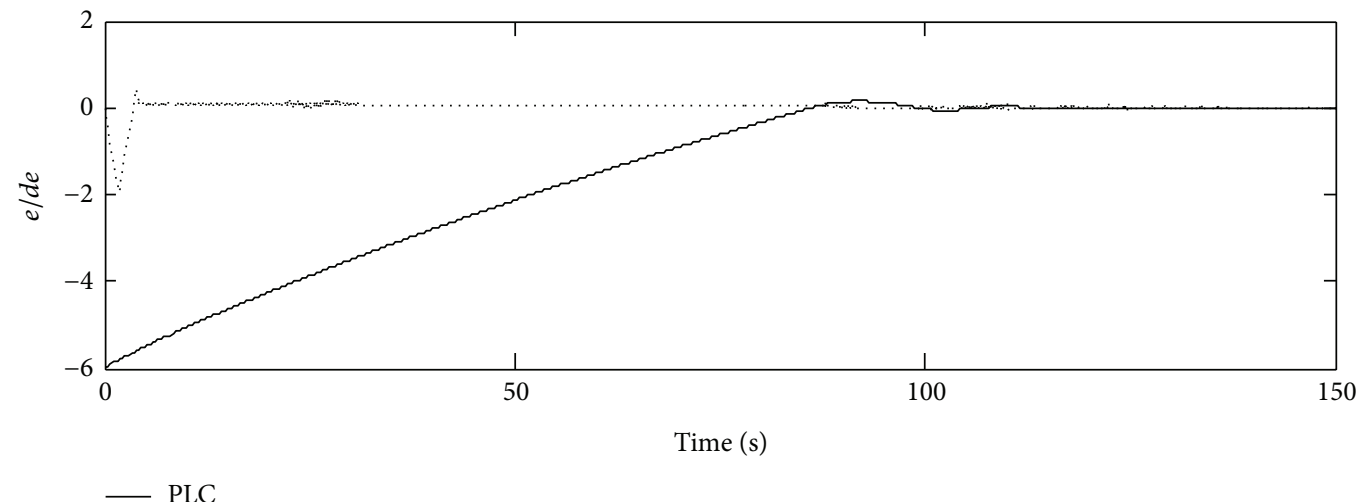

FIGURE 4: Error and its derivative for the different controllers.

A sufficient condition for convergence is given as

$$
\alpha K_{m}-C>\frac{\beta^{2}}{2} \text {. }
$$

\section{Simulation Results and Discussion}

The characteristics of dynamic model of the coupled tanks system are presented in Table 1 [8]. The control input is restricted to be between $u_{\min }=0$ and $u_{\max }=50\left[\mathrm{~cm}^{3} / \mathrm{s}\right]$. The computer simulations are performed using a time interval of $[0: 150] \mathrm{s}$. The parameters of the controller are optimized using a step input with a final value of $6 \mathrm{~cm}$ for the water level. For the other tested water levels no more adjustment of the controllers parameters is performed. Through extensive simulation runs, the optimum controllers' parameters for the different controllers tested in this study are reported in Table 2.

Figure 3 shows the regulation performance for the tested controllers for a desired level of $6 \mathrm{~cm}$. Based on the figure, it can be concluded that the controllers regulate the water 

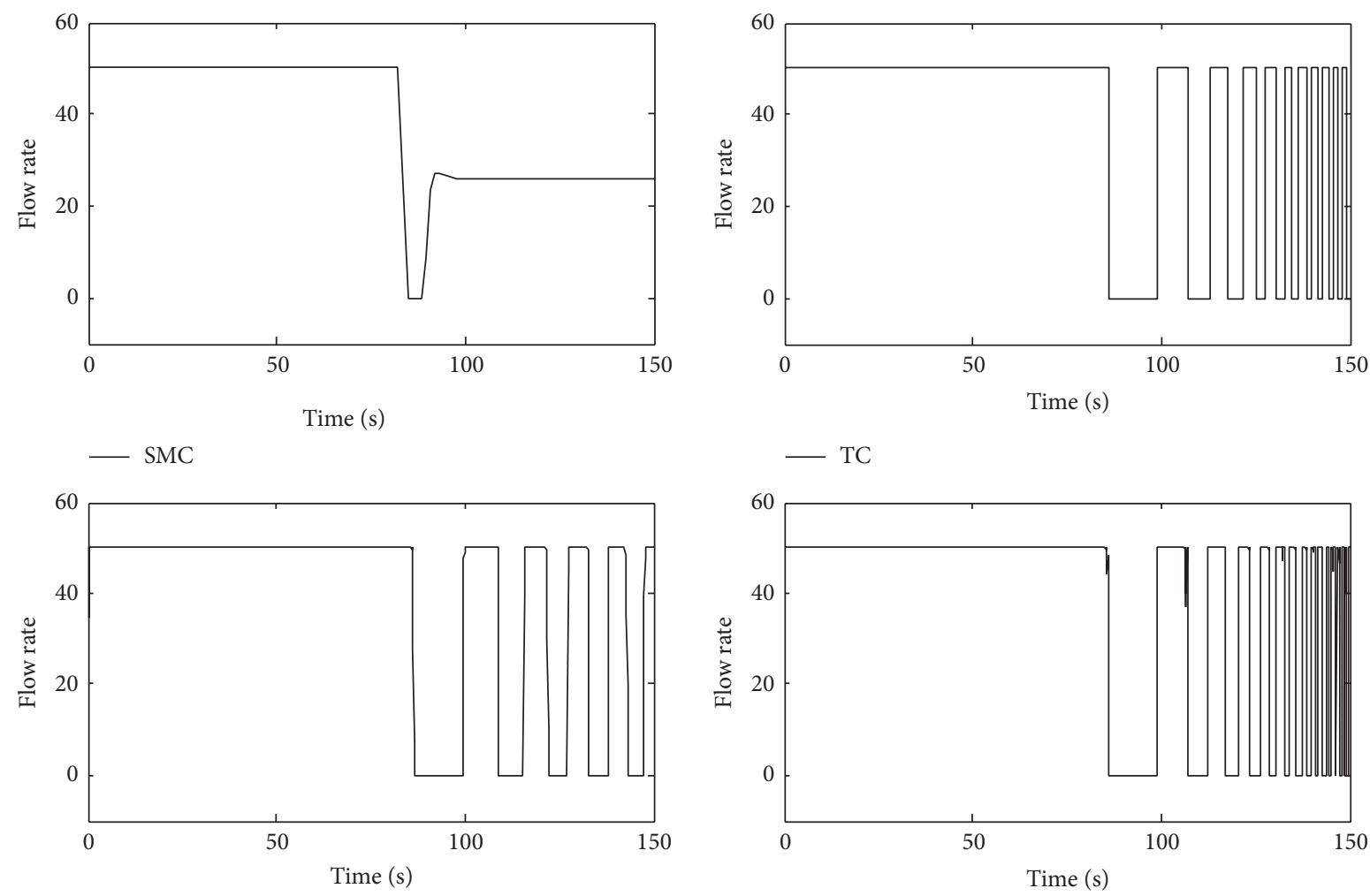

— STC

- QCC

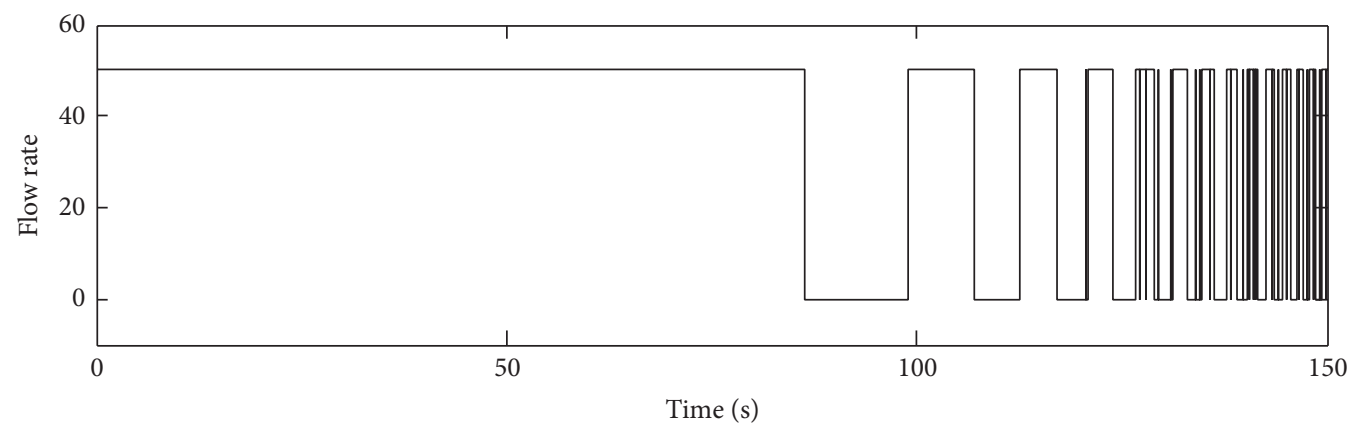

PCL

FIGURE 5: The control signals of the designed controllers.

TABLE 1: Characteristic of the coupled tanks system.

\begin{tabular}{lr}
\hline Gravitational rate $g$ & $981 \mathrm{~cm} / \mathrm{s}^{2}$ \\
Cross-sectional area of both tanks & $208.2 \mathrm{~cm}^{2}$ \\
Area of the connecting pipe $a_{1}$ & $0.58 \mathrm{~cm}^{2}$ \\
Area of the outlet $a_{2}$ & $0.24 \mathrm{~cm}^{2}$ \\
\hline
\end{tabular}

level successfully with approximately the same performance. Note that the controllers are able to regulate any desired water levels without adjustments of any parameter.

The behaviors of sliding surfaces/error are represented in Figure 4 for the desired level of $6 \mathrm{~cm}$. Based on the figure it can be seen that, in all cases, the controllers show typical sliding mode behavior; that is, the error reaches 0 in finite
TABLE 2: Controllers parameters.

\begin{tabular}{lcccccccccc}
\hline Controller & \multicolumn{2}{c}{ SMC } & \multicolumn{2}{c}{ TC } & \multicolumn{2}{c}{ STC } & \multicolumn{2}{c}{ QCC } & \multicolumn{2}{c}{ PCL } \\
\hline Parameters & $\lambda$ & $k$ & $r_{1}$ & $r_{2}$ & $\alpha$ & $\lambda$ & $\alpha$ & $\beta$ & $\alpha$ & $\beta$ \\
\hline Value & 0.67 & 90 & 500 & 100 & 220 & 14 & 65 & 2 & 100 & 1 \\
\hline
\end{tabular}

time and stays 0 afterwards. The derivative of error with respect to time is also shown for the TA, QCC, and PLC controllers. The error derivative also converges to zero. Note that in the case of the 1-SMC and STC the error derivatives are not shown because the error derivatives do not play a major role in the design of the controller.

Figure 5 shows the control signal of the five controllers. The controllers have the same control signal until they reach 
TABLE 3: Time domain performance measures for different $H$ values.

\begin{tabular}{lccccc}
\hline$H(\mathrm{~cm})$ & Controller & $T_{r}(\mathrm{~s})$ & $T_{s}(\mathrm{~s})$ & Os (\%) & IAE \\
\hline \multirow{6}{*}{3.00} & SMC & 29.975 & 36.626 & 1.776 & 52.459 \\
& TA & 30.010 & 51.783 & 7.506 & 54.823 \\
& STC & 30.017 & 52.124 & 7.769 & 56.214 \\
& QCC & 30.010 & 51.250 & 7.085 & 54.580 \\
& PCL & 30.010 & 51.576 & 7.344 & 54.704 \\
\hline \multirow{6}{*}{6.00} & SMC & 69.308 & 84.162 & 0.097 & 234.125 \\
& TA & 69.335 & 95.179 & 2.522 & 236.066 \\
& STC & 69.330 & 95.560 & 2.621 & 236.735 \\
& QCC & 69.335 & 94.630 & 2.393 & 235.909 \\
& PCL & 69.335 & 94.975 & 2.473 & 235.973 \\
\hline \multirow{6}{*}{8.00} & SMC & 101.366 & 123.310 & 0.033 & 445.981 \\
& TA & 101.384 & 123.330 & 1.392 & 447.400 \\
& STC & 101.378 & 123.315 & 1.457 & 447.537 \\
& QCC & 101.384 & 123.330 & 1.327 & 447.329 \\
& PCL & 101.384 & 123.330 & 1.367 & 447.357 \\
\hline
\end{tabular}

the sliding surface. The difference between the signals of the 2-SM controllers is the increase of the on-off control in the order STC and TA, for the QCC and PCL, respectively.

To compare the performance of the controllers, the time domain performance measures such as the settling time, the rise time, the percentage overshoot, and the integral absolute error (IAE) are used. The settling time is defined as the time required for the response to settle within $1 \%$ of the steady state value. The rise time is defined as the time required for the output to change from $10 \%$ to $90 \%$ of its final value. The IAE is given by the following equation:

$$
\mathrm{IAE}=\int_{0}^{t}|\sigma| d t
$$

The performance measures have been computed for three different specified levels of 3,6 , and $8 \mathrm{~cm}$ as listed in Table 3 . It can be seen from the table that all the used controllers have approximately the same rise time in all the cases. The QCC controller has a slightly less settling, overshoot, and IAE.

The results of the tracking test of the five controllers using a square signal reference input are shown in Figure 6. Based on the figure, it can be observed that the tracking performance for the all the controller is good. In terms of the IAE, the values obtained are listed in Table 4 . From the values given in the Table 4, it can be seen that the smallest IAE is for the 1-SM controller; the 2-SMC have approximately the same IAE. From the comparison, it can be reported that the 1-SM controller slightly outperforms the 2-SM controller in all aspects but the controller requires more information than the 2-SM controllers as can be observed from Section 4 .

\section{Conclusions}

In this paper, four second order sliding mode controllers, namely, the twisting, the super twisting, the quasi-con-
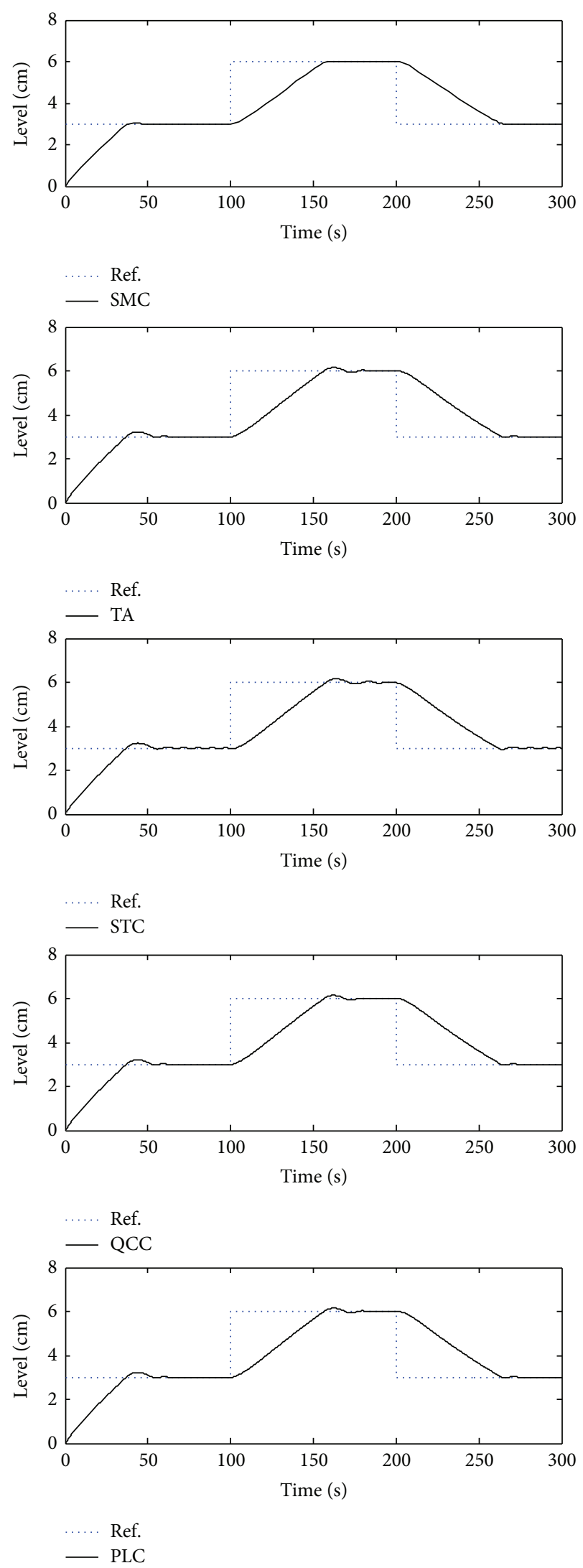

FIGURE 6: Level tracking test for the different controllers. 
TABLE 4: IAE for the different controllers from the tracking test.

\begin{tabular}{lccccc}
\hline Controller & SMC & TA & STC & QCC & PCL \\
\hline IAE & 242.32 & 248.00 & 247.51 & 246.89 & 248.42 \\
\hline
\end{tabular}

tinuous, and the prescribed convergence law controller, have been successfully designed to regulate the water level in the second tank of a coupled tanks system. The efficacy and usability of the proposed controllers are verified through computer simulation tests. Comparison between the controllers is based on the time domain performance measures. Results showed that all the controllers are able to regulate the water level without major differences in their performance. Comparison of the 2-SM controllers against 1-SM controller showed that less information is required in the case of the 2SM controller than the 1-SMC.

\section{Conflict of Interests}

The authors declare that there is no conflict of interests regarding the publication of this paper.

\section{Acknowledgment}

This project was financially supported by King Saud University, Vice Deanship of Research Chairs.

\section{References}

[1] S. K. Singh, N. Katal, and S. G. Modani, "Multi-objective optimization of PID controller for coupled-tank liquid-level control system using genetic algorithm," in Proceedings of the Second International Conference on Soft Computing for Problem Solving (SocProS 2012), December 28-30, 2012, vol. 236 of Advances in Intelligent Systems and Computing, pp. 59-66, Springer, New Delhi, India, 2014.

[2] J.-X. Xu, C.-C. Hang, and C. Liu, "Parallel structure and tuning of a fuzzy PID controller," Automatica, vol. 36, no. 5, pp. 673684, 2000.

[3] N. K. Poulsen, B. Kouvaritakis, and M. Cannon, "Nonlinear constrained predictive control applied to a coupled-tanks apparatus," IEE Proceedings: Control Theory and Applications, vol. 148, no. 1, pp. 17-24, 2001.

[4] A. Kumar, M. Vashishth, and L. Rai, "Liquid level control of coupled tank system using fractional PID controller," International Journal of Emerging Trends in Electrical and Electronics, vol. 3, no. 1, 2013.

[5] V. Utkin, J. Guldner, and M. Shijun, Sliding Mode Control in Electro-Mechanical Systems, vol. 34, CRC Press, 1999.

[6] C. Edwards and S. Spurgeon, Sliding Mode Control: Theory and Applications, CRC Press, 1998.

[7] K. D. Young, V. I. Utkin, and Ü. Özgüner, "A control engineer's guide to sliding mode control," IEEE Transactions on Control Systems Technology, vol. 7, no. 3, pp. 328-342, 1999.

[8] N. B. Almutairi and M. Zribi, "Sliding mode control of coupled tanks," Mechatronics, vol. 16, no. 7, pp. 427-441, 2006.

[9] S. Aydin and S. Tokat, "Sliding mode control of a coupled tank system with a state varying sliding surface parameter," in Proceedings of the IEEE International Workshop on Variable Structure Systems (VSS '08), pp. 355-360, IEEE, Antalya, Turkey, June 2008.

[10] A. Boubakir, F. Boudjema, and S. Labiod, "A neuro-fuzzysliding mode controller using nonlinear sliding surface applied to the coupled tanks system," International Journal of Automation and Computing, vol. 6, no. 1, pp. 72-80, 2009.

[11] M. K. Khan and S. K. Spurgeon, "Robust MIMO water level control in interconnected twin-tanks using second order sliding mode control," Control Engineering Practice, vol. 14, no. 4, pp. 375-386, 2006.

[12] C. Pukdeboon, "Second-order sliding mode controllers for spacecraft relative translation," Applied Mathematical Sciences, vol. 6, no. 100, pp. 4965-4979, 2012.

[13] İ. Eker, "Second-order sliding mode control with experimental application," ISA Transactions, vol. 49, no. 3, pp. 394-405, 2010.

[14] S. Mondal and C. Mahanta, "Composite nonlinear feedback based discrete integral sliding mode controller for uncertain systems," Communications in Nonlinear Science and Numerical Simulation, vol. 17, no. 3, pp. 1320-1331, 2012.

[15] S. Mondal and C. Mahanta, "Nonlinear sliding surface based second order sliding mode controller for uncertain linear systems," Communications in Nonlinear Science and Numerical Simulation, vol. 16, no. 9, pp. 3760-3769, 2011.

[16] S. Mondal and C. Mahanta, "A fast converging robust controller using adaptive second order sliding mode," ISA Transactions, vol. 51, no. 6, pp. 713-721, 2012.

[17] M. Defoort, T. Floquet, A. Kokosy, and W. Perruquetti, "A novel higher order sliding mode control scheme," Systems \& Control Letters, vol. 58, no. 2, pp. 102-108, 2009.

[18] A. Levant, "Higher-order sliding modes, differentiation and output-feedback control," International Journal of Control, vol. 76, no. 9-10, pp. 924-941, 2003.

[19] Y. Shtessel, C. Edwards, L. Fridman, and A. Levant, Sliding Mode Control and Observation, Birkhäuser, 1st edition, 2014.

[20] M. K. Khan, S. K. Spurgeon, and A. Levant, "Simple output feedback 2-sliding controller for systems of relative degree two," in Proceedings of the European Control Conference (ECC '03), vol. 3, September 2003.

[21] A. Levant, "Quasi-continuous high-order sliding-mode controllers," IEEE Transactions on Automatic Control, vol. 50, no. 11, pp. 1812-1816, 2005. 


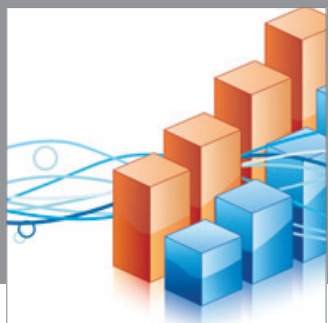

Advances in

Operations Research

mansans

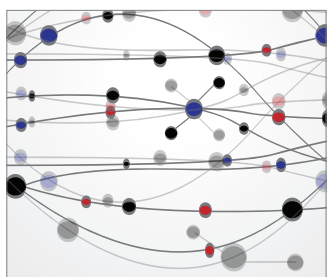

The Scientific World Journal
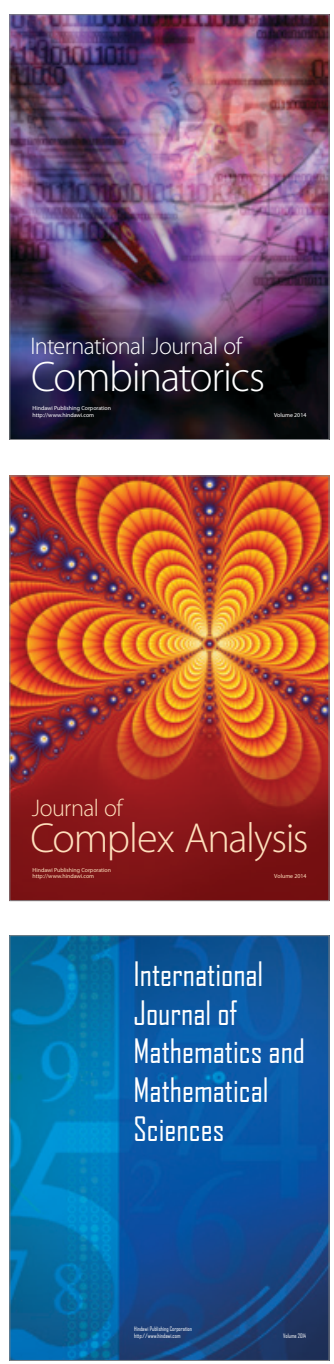
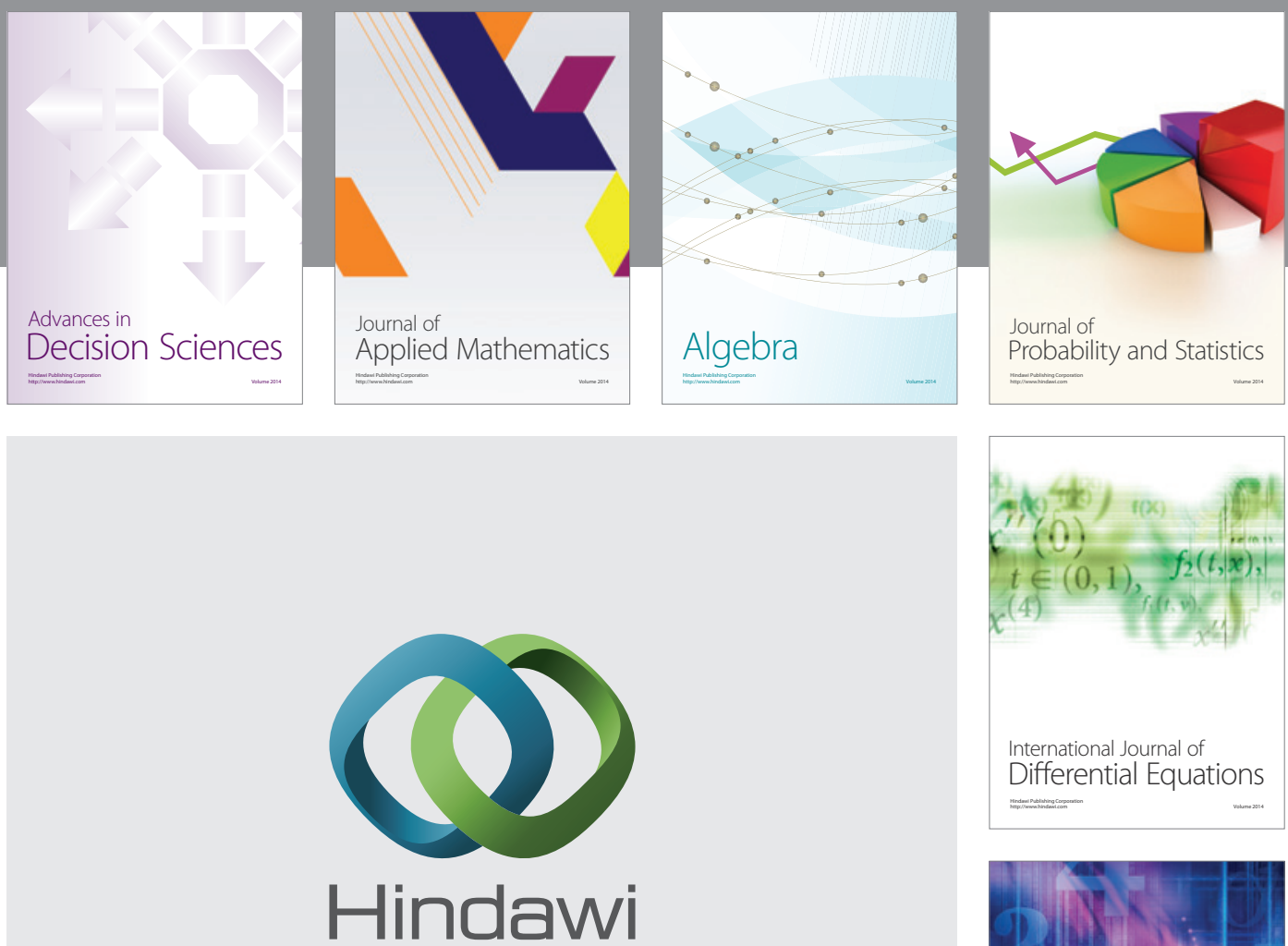

Submit your manuscripts at http://www.hindawi.com
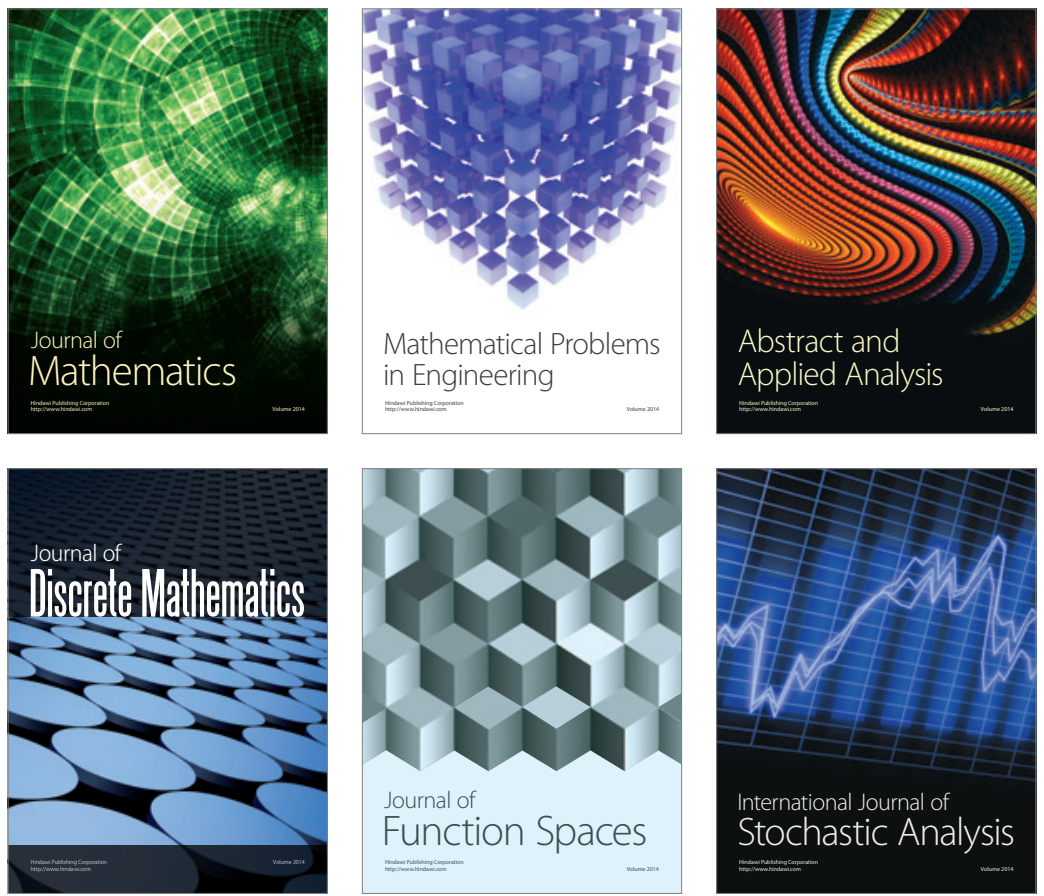

Journal of

Function Spaces

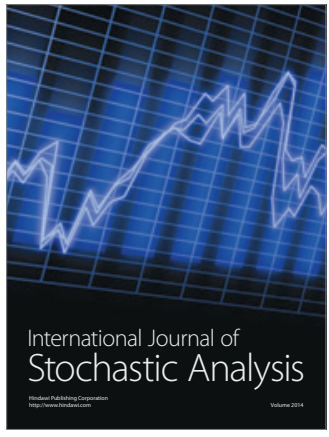

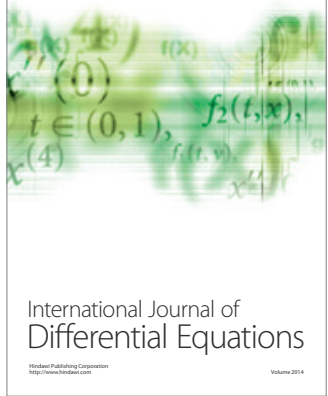
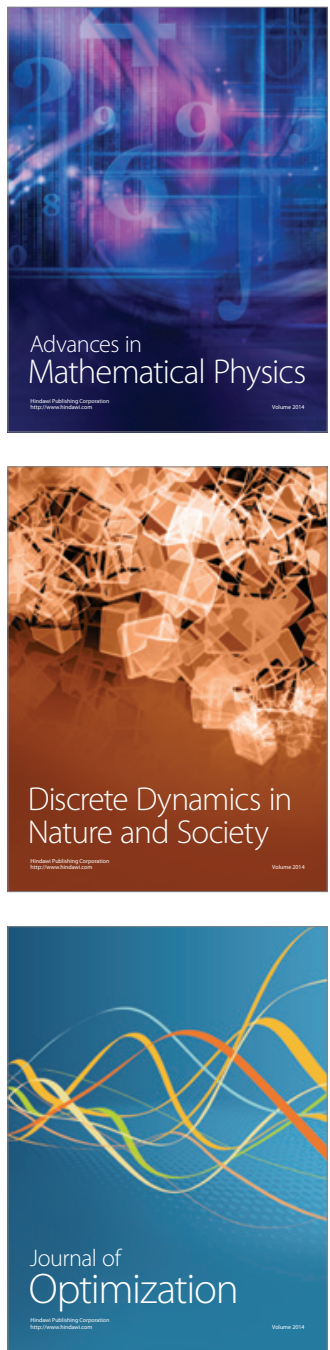